\title{
Early detection of damage to nerves in leprosy
}

\author{
D D PALANDE \& R E M BOWDEN \\ Department of Pathology, Royal College of Surgeons, Lincoln's Inn \\ Fields, London WC2
}

\section{Accepted for publication 18 September 1991}

\begin{abstract}
Summary Methods of examining and diagnosing damage to nerves commonly involved in leprosy are described. The equipment used is inexpensive, gives reliable and repeatable results and is useful in making objective assessments in terms of function in everyday living.
\end{abstract}

\section{Introduction}

The severe disability and disfigurement resulting from the destruction of tissues by leprosy is exacerbated by the involvement of nerves. Early signs of neural involvement may be insidious and overlooked, but prompt detection and vigorous treatment of the disease in its reactional stage are essential if it is to be cured and disability avoided or minimized.

In the majority of cases there is no spontaneous pain' ${ }^{1}$ although numbness and tingling may occur. Weakness may be minimal and sensory loss dissociated in the early stages with loss of protective pain preceding loss of other modalities. ${ }^{2}$ Patients may not only be unaware of minor alterations in function, but also may be reluctant to acknowledge them, because they fear isolation and prejudice. The need to publicize the feasibility of correcting deformities and the possibility of cure with early and conscientiously carried out treatment cannot be overemphasized.

Easily performed and rapid field tests of the function of the six nerves most commonly affected in leprosy were described by Fritschi, ${ }^{3}$ who stressed the need for referral to an established centre for more detailed testing. The purpose of this paper is to outline such procedures and to draw attention to anomalies ${ }^{4,5}$ and trick movements which may deceive the experienced and inexperienced.

\section{General considerations}

Thorough examination is simple but time consuming and demands concentration by both the examiner and patient. Patients should be able to sit or lie down in comfort in a quiet and well-lit room. For bare-footed patients washing the feet is not only more comfortable, but also softens the skin. Patients should not be hungry, thirsty, or in need of the toilet. 
Ample time must be allowed for explanations and for listening to the patient's history and progress.

\section{EQUIPMENT}

Inexpensive and reliable equipment for testing the thresholds of modalities of touch, and pain, is illustrated in Figure 1. Discrimination of textures can be assessed by using standard graded sandpaper; two-point discrimination can be measured with fine calipers and a steel rule marked in centimetres and millimetres. Testing for deep pain by needles is inappropriate, especially on the feet if the patient is habitually bare footed. Deep sensation might be tested by asking patients to walk on special mats covered with smooth pebbles of graded sizes, or by the use of calibrated pressure recording appliances. Battery operated pocket apparatus for testing thermal sensibility has been described by Srinivasan \& Strumpe. ${ }^{2}$ Intensities and rates of vibration can be measured either with a vibrometer, ${ }^{6}$ or else tuning forks can be used. Simple electrical stimulation demonstrates the presence or absence of nerve conduction and is valuable in detecting anomalous innervation and the block to conduction in early involvement of nerves. ${ }^{7}$ Precise measurements of rates of nerve conduction and electronmyography are objective tests and are desirable for critical assessment and research in specialized centres.

\section{RECORDS}

The history should include the patient's age, sex, occupation, family history and possible contacts who should be traced, examined, and followed up. Symptoms should be described in the patient's own words. If they are inarticulate from fear or lack of education, questions should be helpful, but never direct, e.g. 'Is your hand as strong as it always was, or is it weaker?' is preferable to 'Have you noticed any weakness?' It is particularly important to avoid influencing suggestible individuals when discussing early sensory symptoms and signs of motor dysfunction.

A well-thought-out protocol is essential for assessing progress and planning reconstructive surgery. The power of individual muscles ${ }^{8}$ should be graded and recorded on charts. The Medical Research Council scale is useful and simple to apply:

0 , complete paralysis; 1 , flicker of contraction but no movement; 2 , movement of joint with gravity eliminated; 3 , movement against gravity but not resistance; 4 , movement against gravity and resistance, but weaker than normal; and 5, indistinguishable from normal.

From the practical point of daily living, manipulation of test objects such as those illustrated in Figures 2 and 3 is useful ${ }^{9-11}$ and is helpf ul in rehabilitation since the optimum size of handles for tools can be found for each individual. In every case, contralateral parts of the body should be tested. ${ }^{6}$ Sensory loss or abnormality can be mapped, and in the absence of photographs, indicated on dated charts. Tests should be demonstrated first on the examiner's own hand, then on a normal part of the patient's body whilst his eyes are open. The suspected and contralateral areas are examined with the patient's eyes closed or shielded. Allow frequent short pauses for relaxation.

Autonomic function can be recorded either qualitatively or quantitatively by noting the colour, temperature and moisture of skin. Skin resistance is measured by a 

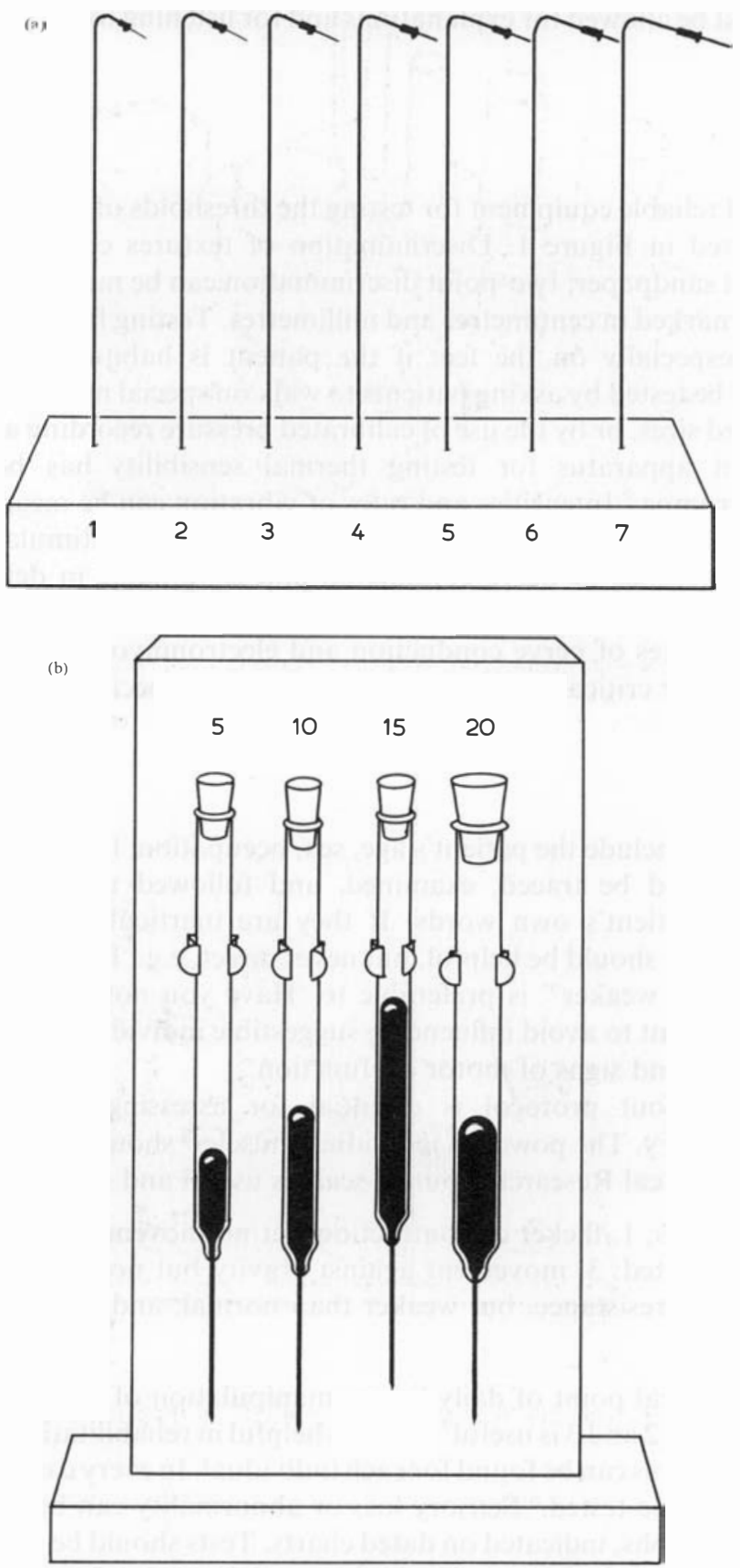

Figure 1. (a) Home-made $v$ s Frey hairs (as modified by the late $G$ Weddell). These graded suture materials are mounted on spokes of bicycle wheels which are angulated. $1 \mathrm{~cm}$ lengths project freely. To conform with previously recorded work ${ }^{18}$ No. 3 should be equivalent to 1 g pressure. Other grades should be noted and calibrated on an accurate balance and replaced at intervals. (b) Cutting needles are mounted on weights of 5, 10, 15 and $20 \mathrm{~g}$ which run freely, either in glass tubing, or better still, rigid polythene tubes. 


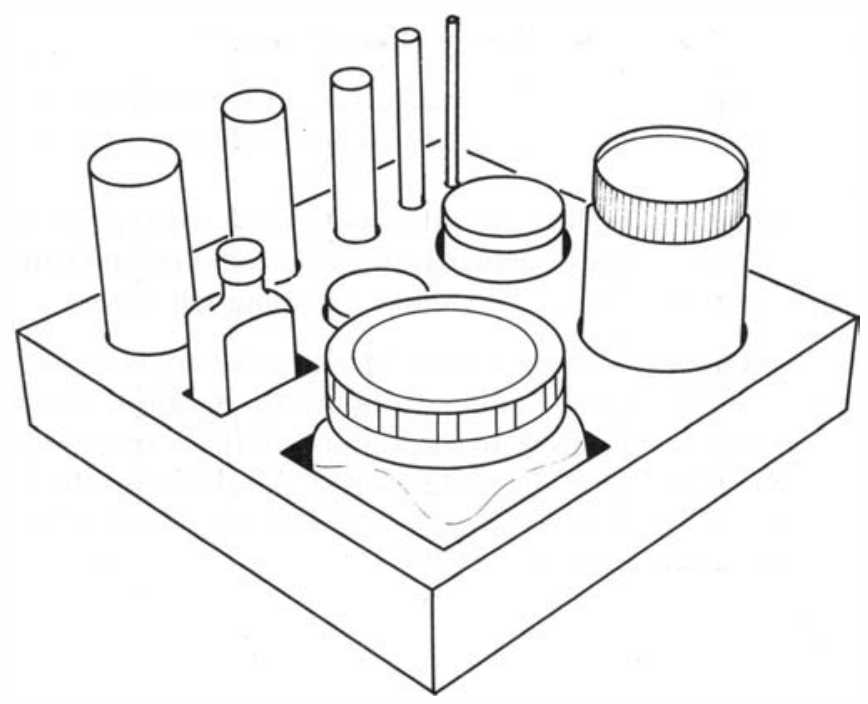

Figure 2. Home-made equipment, wooden rods of different diameters, test the optimum size of handles for tools, also timed taking and replacing in the slots can give semi-objective test of dexterity. Square jars in square holes are useful for patients who are either one-handed, or who have very weak hands.

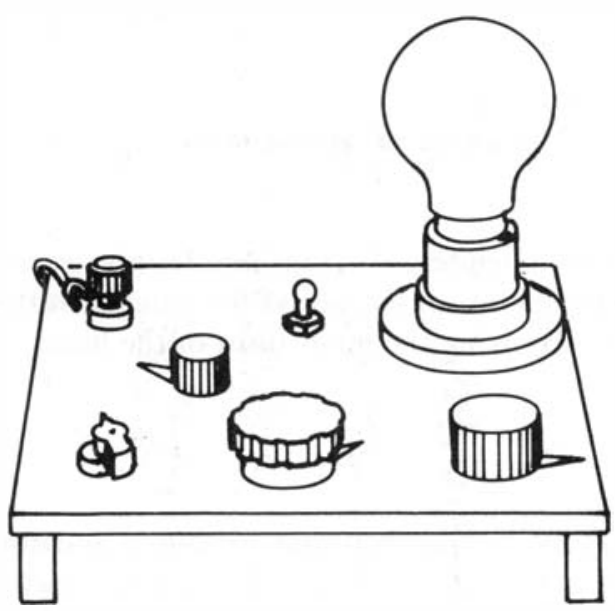

Figure 3. Light bulbs require precision for engaging lugs, power for seating in sockets. The knobs and switches require precise manipulation.

dermometre which is a simple and inexpensive instrument consisting of an ohmmeter, an indifferent electrode and a mapping one with a head of $\frac{1}{2}$ to $1 \mathrm{~cm}$ diameter. In hot climates sweating is often visible and there is no need for ninhydrin or quinizarin tests. It can be enhanced by application of a sphygomamometer, inflating the cuff above systolic pressure for $1-2$ min. $^{12}$ 


\section{EARLY SYMPTOMS AND SIGNS OF, NEURAL INVOLVEMENT}

Spontaneous discomfort or pain may be aggravated by movement of related joints or muscles, and there may be localized tenderness and swelling of the nerve and referred paraesthesia.

2 Partial and developing sensory loss are often associated with spontaneous tingling in the distribution of the nerve 'like insects crawling over the skin' and with hyperaesthesia which feels 'uncomfortable', 'nasty' or 'unlike anything felt before'.

Parts of limbs may feel heavy or even absent. Movement may become clumsy, and the subject became tired too quickly, or suffer from cramp. Cramp, occasionally spontaneous, may waken a patient from sleep. In the lower limb there are alterations in gait and weight bearing. ${ }^{13,14}$ Weakness and fully developed paralysis results in characteristic paralytic deformity due to imbalanced activity of unaffected muscles. An early sign is the inability to sustain an end position.

\section{OBJECTIVE CHANGES IN SKIN}

These are varied; there may be altered pigmentation; transient hyperhydrosis, hypo- or anhydrosis; altered colour and temperature due to vasomotor disturbance. In longestablished cases the pulps of fingers and toes may atrophy, with alterations in curvature and thickness of nails and flattening and blurring of papillary ridges. Loss of sensation increases the risks of painless ulceration, burns and secondary infection with a devastating loss of tissue.

\section{Early symptoms and signs of damage to specific nerves}

\section{THE ULNAR NERVE}

Damage, with or without a tender swelling, commonly occurs at or just above the posteromedial aspect of the elbow, or just above the anterior surface of the wrist, or in the dorsal cutaneous branch supplying the inner third of the hand, the little finger, and half the ring finger.

\section{History}

With high lesions, sensory disturbance is found on palmar and dorsal surfaces of the inner third of the hand and medial $1 \frac{1}{2}$ digits. Symptoms are increased by the stretching of the nerve, by flexion of the elbow and by compression in the cubital tunnel during active ulnar deviation of the wrist. The dorsal branch escapes in lesions at the wrist. Whatever the level of damage, there is increasing weakness and fatigue of sustained grip and clumsiness of skilled and precise movement of the fingers; implements tend to slip from the hand; turning off taps, wringing out towels and loosening tops of jars become difficult. Clawing of fingers impedes removing things from pockets.

\section{On Examination}

The early paralytic deformity and later wasting of intrinsic muscles of the hand, especially the first dorsal interosseous, are characteristic. Clawing is less pronounced in higher than 


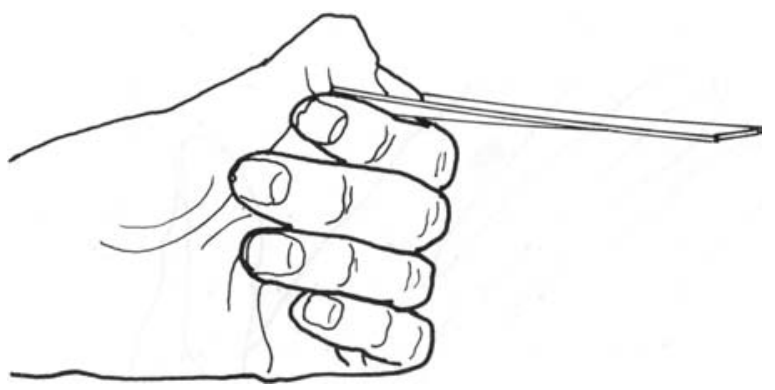

Figure 4. A positive Froment's sign using flexor pollicis longus instead of adductor pollicis.

in lower lesions where flexor digitorum profundus is unaffected. The hypothenar eminence and medial aspect of the thenar eminence overlying adductor pollicis are soft in early paralysis and atrophied later. The distal transverse metacarpal arch is lost, or diminished at rest, and this loss is accentuated when attempting to extend the fingers of the outstretched hand. Active adduction of fingers is lost, but trick abduction is possible due to the pull of divergent tendons of the long extensors. The paralytic abduction deformity of the little finger is one of the earliest signs to appear and the last to disappear in recovery as the small fourth palmar interosseous has to counteract the long flexors and extensors and abductor minimi digiti. Adduction is tested by slipping a sheet of paper between fingers and asking the patient to resist when it is pulled away. Loss of adductor pollicis can be compensated by trick flexion of flexor pollicis longus (Figure 4). Straight finger flexion at the metacarpophalangeal joints is only possible with intact ulnar intrinsic muscles (Figure 5).

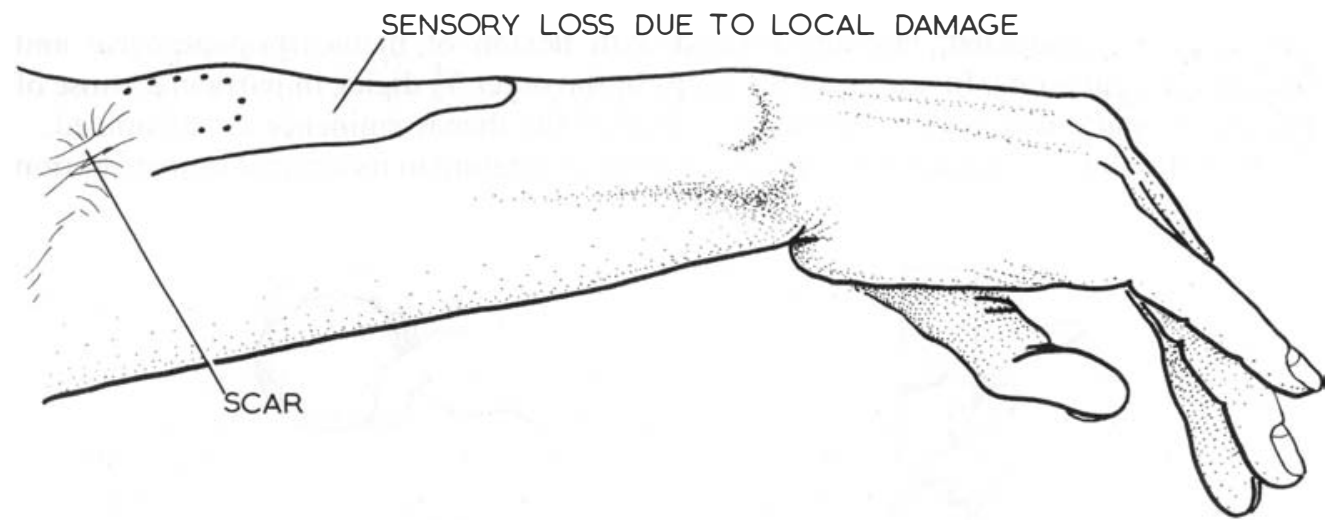

Figure 5. Paralysis of posterior interosseous branch of the radial nerve; note persistent extension of interphalangeal joints and inability to extend metacarpophalangeal joints.

THE MEDIAN NERVE

The common site of damage is just above the wrist crease. 


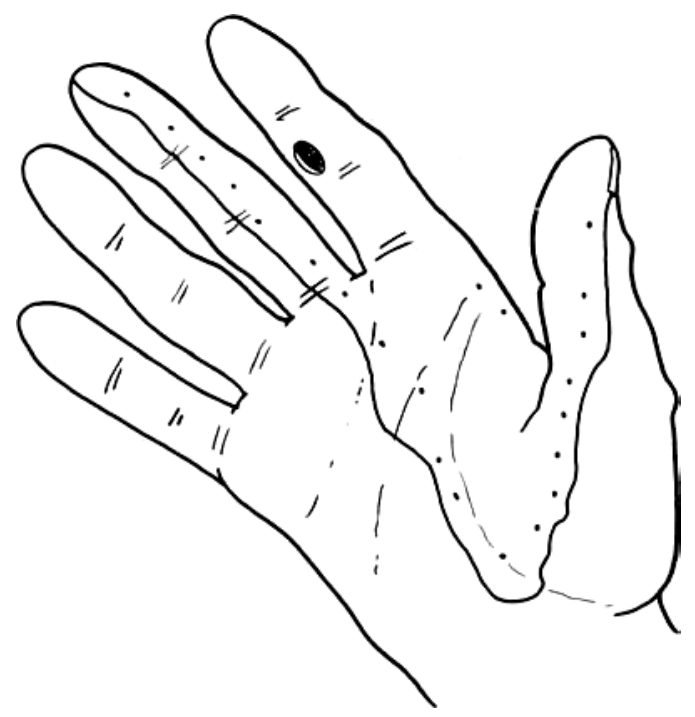

Figure 6. Anomalous sensory supply with a complete division of the median nerve; note the deep painless burn.

\section{History}

In such low lesions knocks and chaffing by coat cuffs can produce tingling in the hand and outer $3 \frac{1}{2}$ digits. Increasing difficulty is experienced in picking up small objects, doing up buttons, holding a pen or small tool, putting keys into locks, threading needles, and recognizing coins in pockets.

\section{On examination}

The thumb is adducted, laterally rotated with flexion of metacarpophalangeal and interphalangeal joints. In late cases the pulps of the outer $3 \frac{1}{2}$ digits, in particular those of the thumb and index finger, and the outer half of the thenar eminence are atrophied.

Palpation of the swollen nerve produces pain or tingling in its cutaneous distribution

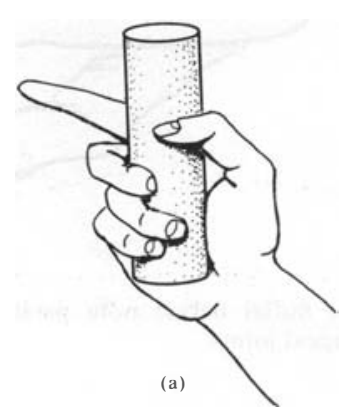

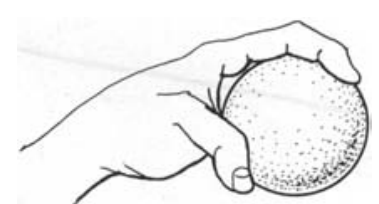

(b)

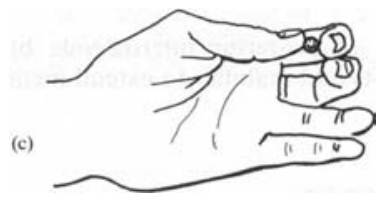

Figure 7. (a) Power grip with a high median lesion. (b) and (c) Variants of precision grip with a median lesion at the wrist. 
in which sensation is either diminished or absent. Anomalous motor and sensory supply by median and ulnar nerves must be borne in mind $^{4}$ (Figure 6). Even with anomalous innervation, it is impossible to sustain abduction of the thumb at right angles to the palm and a pulp-to-pulp grip between the index finger and thumb is impossible (Figure 7).

\section{RADIAL NERVE}

Power and precision grips are weakened as radial extensors fail to stabilize the wrist. ${ }^{9,11}$ Extension of the metacarpophalangeal joints is weak or lost, although extension of interphalangeal joints persists, provided the ulnar nerve is intact. The area of loss of cutaneous sensibility is notoriously variable (Figure 8(a) and (b)) and may be important if the back of the hand is subjected to knocks at work, e.g. harvesting by hand with a sickle.
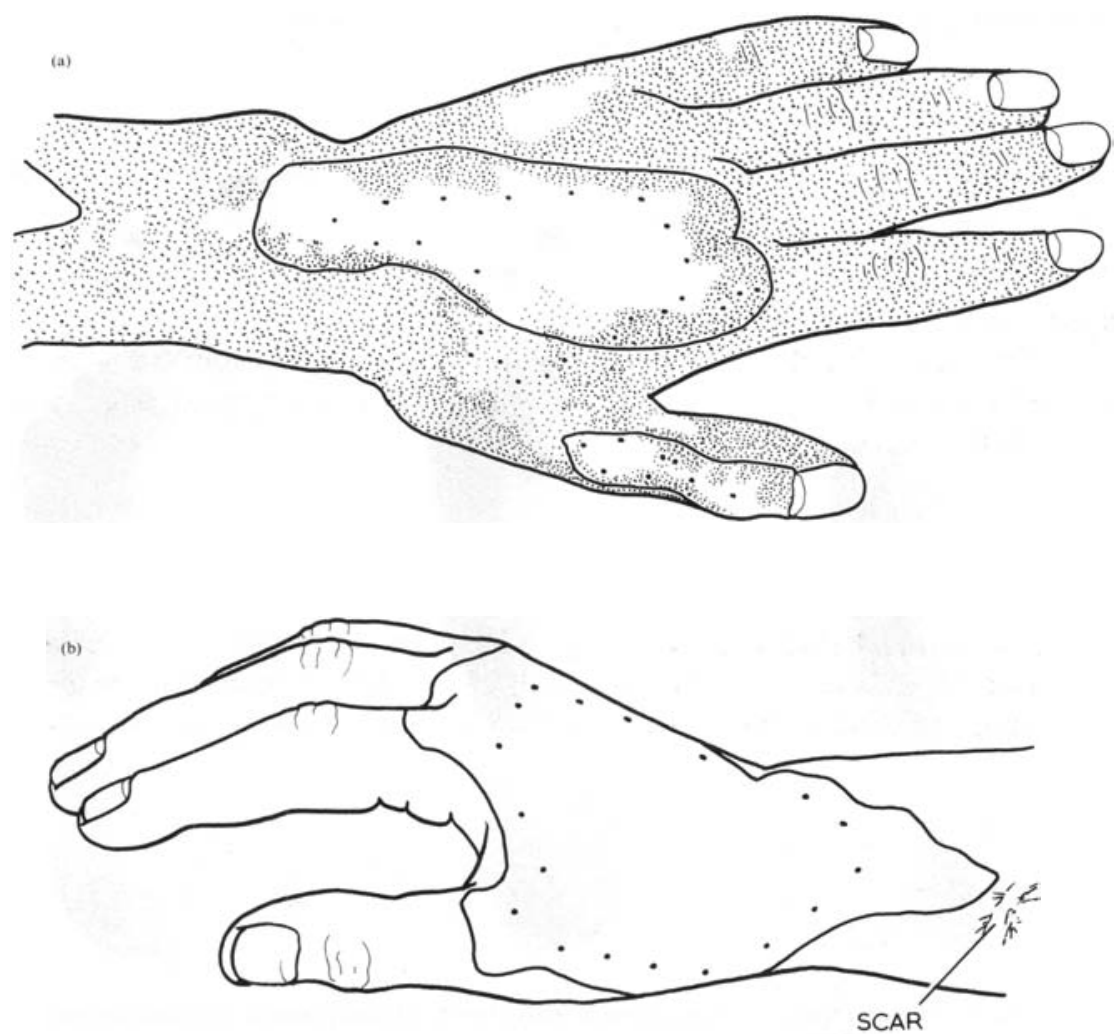

Figure 8. (a) Quinizarin sweating test and map of anaesthesia to $1 \mathrm{~g}$ vs Frey hair, —_; analgesia to light pen prick, $\cdots$ a ter complete section of radial nerve. (b) Sensory loss mapped as in Figure 10(a) af ter section of the superficial radial nerve.

THE COMMON PERONEAL NERVE

The common peroneal nerve is usually affected in the region of the neck of the fibula. 


\section{History}

The patient may 'catch his foot' or 'turn the ankle' on both flat and rough surfaces due to foot drop and loss of the evertors of the foot.

\section{On examination}

In advanced cases the anterolateral aspect of the leg and extensor brevis digitorum are wasted. Callosities may develop under the 5th metatarsal head if the unopposed action of tibialis posterior is not corrected by an appropriate 'lively' splint. There is weakness or loss of dorsiflexion of the ankle and metatarsophalangeal joints. If no active splint is used there will also be a contracture of the calf muscle and disturbance of weight bearing and gait that are reflected in footprints (Figure 9), wear of shoes and incidence of callosities (Figure 10(a). Loss or altered sensitivity is found on the lateral aspect of the leg and dorsum of the foot.
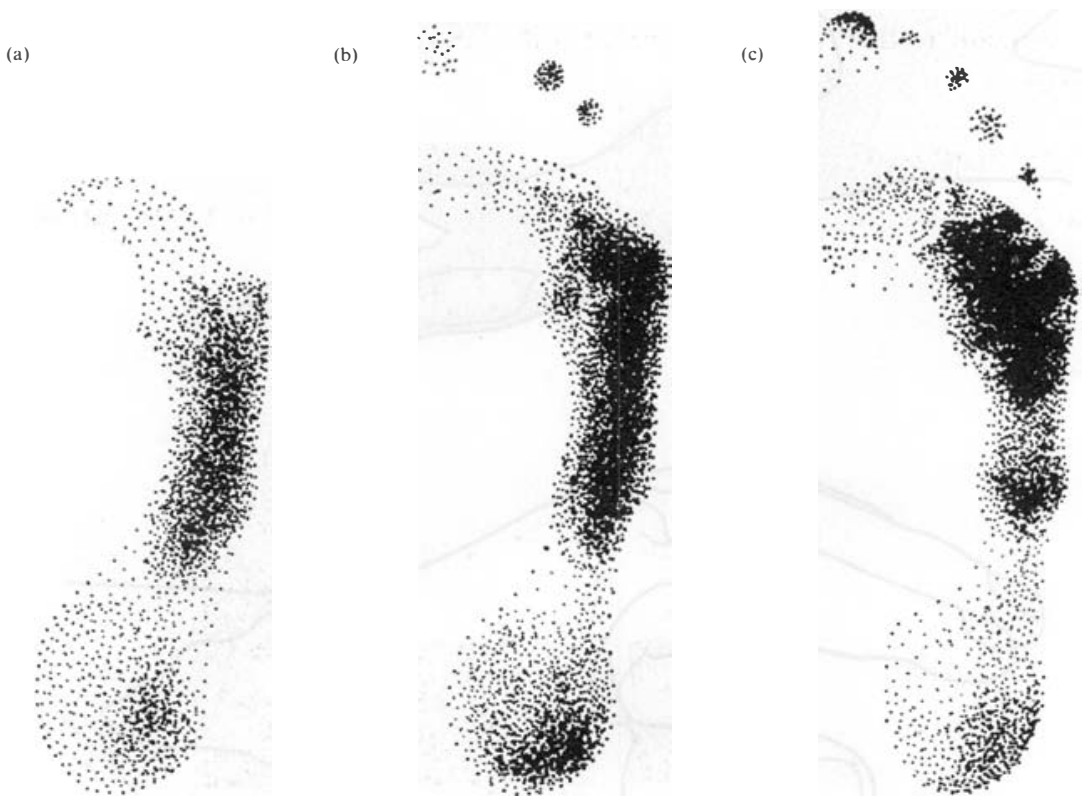

Figure 9. Footprints of patient with common peroneal palsy (without lively splint). (a) standing on both feet; (b) standing on affected foot; (c) walking print.

\section{THE TIBIAL AND SURAL NERVES}

The tibial nerve is commonly involved in the medial retromallealar fossa and the sural in its long subcutaneous course from mid-calf, behind the lateral malleolus and along the lateral border of the foot. 


\section{History}

Patients who wear shoes may notice increasing clawing of toes with painful callosities over the proximal interphalangeal joints. Bare-footed patients may have painless ulcers; sometimes the first symptom may be painful lymph nodes in the vertical inguinal group. If patients sit cross-legged, ulceration is also common on the side of the 5th metatarsal head in the area supplied by the sural nerve.

\section{On examination}

The clawing of toes is marked, and callosities may be found under all metatarsal heads (Figure 10(b)). The transverse metatarsal arch is flattened, and ulceration is common under the 1st metatarsal head due to the pressure of toeing off with a grinding movement in which eversion is involved. The intrinsic muscles on the sole are soft or, in longstanding cases, atrophied. Inability to pick up crumpled paper is an early sign of weakness of intrinsic muscles. Weak toe spreading is possible by the pull of divergent tendons of the long extensors and flexors. Loss of sensation is serious as it involves the weight bearing of the foot and the lateral border of the foot if the sural nerve is affected.

As in lesions of the peroneal, the normal pattern and rhythm of gait are lost, and weight bearing is disturbed with consequent abnormal wear of footwear ${ }^{12-14}$ (Figure 10(b)-(d)).

\section{TRIGEMINAL NERVE}

Corneal sensitivity must be tested, using a wisp of cotton wool. Diminution or loss are
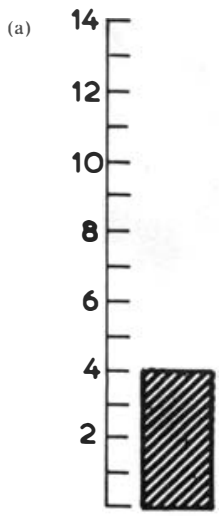

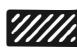

2

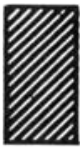

3
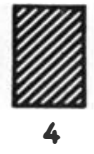

5

(b)
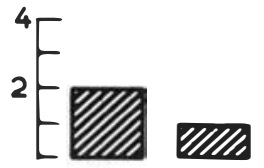

2

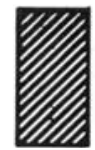

3

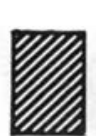

4

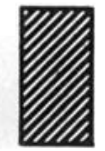

5

METATARSAL HEADS

Figure 10 (a) Incidence and distribution of calosities in 24 patients with paralysis of the common peroneal nerve and inadequate correction of foot drop. (b) Incidence and distribution of calosities in 14 patients with tibial nerve paralysis (note the foot falls into inversion during foot strike because of the structure of the talus). 

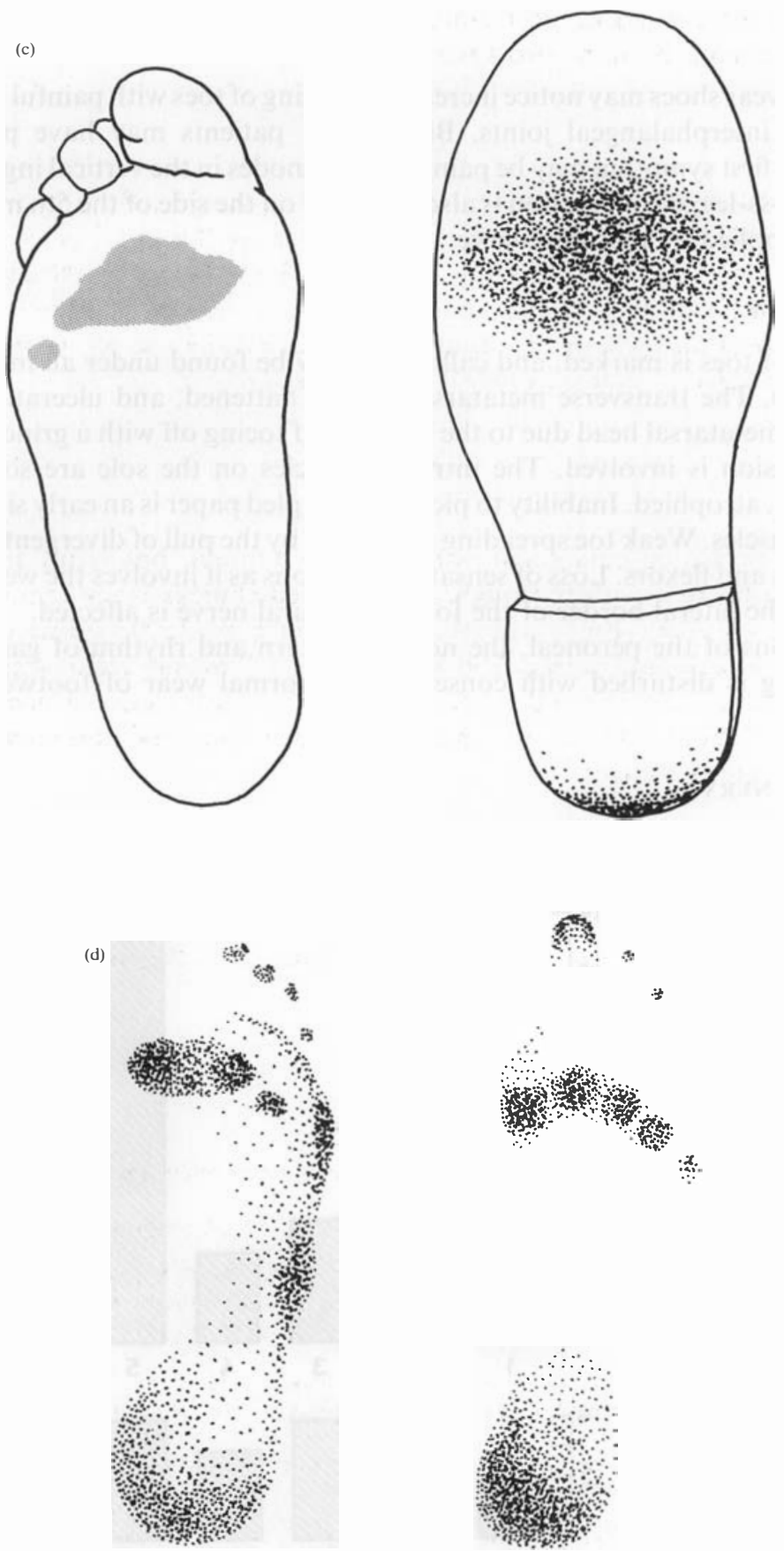

Figure 10 (c) Foot of patient with paralysed intrinsic muscles showing calosities. Shoe wear is excessive under metatarsal heads. (d) 1 Footprints of two patients with paralysed intrensics. Note wasted foot which increases pressure of weight bearing per sq $\mathrm{cm} ; 2$ Note excessive pressure under metatarsal heads, especially under the first. 
dangerous and there is risk of ulceration, scarring or even rupture followed by escape of aqueous and vitreous humors, prolapsed iris and retinal detachment.

\section{FACIAL NERVE}

Involvement of this cranial nerve also has serious consequences. The disfigurement of paralysis is socially embarrassing, but the danger to the eye ${ }^{15}$ is of overwhelming importance as there is a weakness which leads to the loss of the protective blink reflex and ability to close the eyelids. Emergency treatment is needed, particularly if there is associated corneal anaesthesia. Protective goggles and the use of eyedrops to prevent drying are imperative. Tarsorraphy may be indicated. ${ }^{15}$

\section{Conclusion}

Sophisticated electronic equipment is a valuable adjunct, but is no substitute for a carefully taken history, with thorough and recorded clinical examination. In addition, enlisting active co-operation of patients will minimize incidental formation of contractures, trophic ulcers and burns. The importance of regular follow-up examination, conscientious taking of drugs and wearing corrective and protective appliances cannot be over emphasized. Sound advice prevents unnecessary inactivity, and rehabilitation goes hand in hand with treatment. ${ }^{16.17}$

\section{Acknowledgment}

Thanks are due to Mrs Audrey Besterman for illustrations drawn from photographs of patients, actual equipment and footprints.

\section{References}

1 Srinivasan H, Raokk S, Shanmurngam N. Steroid therapy in recent 'quiet' nerve paralysis. Lepr Rev, 1982; 54: 412-19.

2 Srinivasan H, Stumpe B. Value of thermal sensibility testing in leprosy diagnosis in the field-field trial of a pocket device. Lepr Rev, 1989; 60: 317-26.

3 Fritschi EP. Field detection of early neuritis in leprosy. Lepr Rev, 1987; 58: 173-7.

4 Rowntree T. Anomalous innervation of the hand muscles. J Bone Jt Surg, 1949; 31B: 505-10.

5 Falconer D, Spinner M. Anatomic variations in the motor and sensory supply of the thumb. Clin Orthopedics Related Res, 1985; 195: 83-96.

6 Hammond CJ, Klenerman P. Protective sensation in the foot in leprosy. Lepr Rev, 1988; 59: 347-54.

7 Brand P. In: Leprosy in Theory and Practice. R G Cochrane (ed). John Wright \& Sons Ltd, London 1959; Ch XXI, p. 268.

${ }^{8}$ Brain (The Journal) Aids to the examination of the peripheral nervous system 3rd ed superseding the Med Res Counc War Memoranda 1986. Baillière Tindall, London.

${ }^{9}$ Napier JR. The prehensile movements of the human hand. J Bone Jt Surg, 1956; 38B: 902-13.

${ }^{10}$ Moberg E. Objective methods for determining the functional value of sensibility in the hand. J Bone Jt Surg, 1958; 40B: 454-76.

11 Bowden REM, Napier JR. Assessment of hand function after peripheral nerve injuries. J Bone Jt Surg 1961; 43B: 481-92.

12 Palande DD. Some clinical and laboratory signs indicating external compression of a nerve trunk in leprosy. Details and relations. Le pr Rev, 1976; 47: 35-9. 
13 Barnett CH, Bowden REM, Naper JR. Shoe wear as a means of analysing abnormal gait in males. Ann Phys Med, 1956; 111: 121-42.

14 Duckworth T. Pedography in The foot. Helal B, Wilson D (eds). Churchill Livingstone, Edinburgh, London, Melbourne, New York, 1988; 1, pp. 108-45.

${ }_{15}$ Duck Elder S. Leprosy. In A system of ophthalmology. Henry Kempton, London 1974 X V Ch 2 pp. 111-15.

16 Brand PW. Management of the insensitive limb. Physical Ther, 1979 59: 8-12.

17 Prem Kumar R, Brandsma JW. A method to determine pressure distribution of the hand. Lepr Rev, 1986; 57: 39-43.

18 Medical Research Council. Peripheral nerve injuries. Spec Rep Ser Med Res Counc No. 282, 1954, HMSO, London. 